\title{
Uncovered Interest Parity What can we learn from panel data?
}

\author{
Jörg Breitung and Ralf Brüggemann
}

In this chapter we illustrate the use of XploRe panel data quantlets. We apply some of the panel quantlets to a macroeconomic data set to see whether we find evidence in favor of the uncovered interest parity (UIP). Emphasis is given to the illustration of quantlets rather then on elaborated discussion of theory. We use macroeconomic panel data from 16 OECD countries to investigate the empirical evidence for the UIP. To this end a simple fixed effects model is estimated. We deal with the problem of first order autocorrelation by specifying a dynamic panel data model, which is estimated using a GMM estimation procedure suggested by Arrelano and Bond (1991). Furthermore we demonstrate XploRe's capability to test for unit roots in panel data.

\section{The Uncovered Interest Parity}

Exchange market participants constantly shift funds around the globe such that yields of international assets with similar risk should be equalized. However, sometimes we do observe substantial differences in interest rates across different countries. To get a more complete picture we have to take exchange rates into account. This is done by the theory of uncovered interest parity (UIP). A typical macroeconomic textbook model of the UIP (e.g. Burda and Wyplosz 1993) has the following form: Let us denote the domestic interest rate as $i$, the foreign interest rate as $i^{*}$ and the nominal exchange rate as $S$. At time $t$ we can write the UIP as

$$
(1+i)_{t}=\left(1+i^{*}\right)_{t} \frac{E\left(S_{t+1}\right)}{S_{t}} .
$$

Simply speaking, the foreign interest income expressed in the domestic currency should equal the domestic interest rate. From this relationship we obtain the 
approximation:

$$
i_{t} \simeq i_{t}^{*}+E\left(s_{t+1}\right)-s_{t},
$$

where $s_{t}$ denotes the logarithm of $S_{t}$, i.e. $s_{t}=\log S_{t}$.

Following MacDonald and Nagayasu (1999) we modify equation (2). First, we use real exchange rates and real interest rates. Second, since it is not clear whether the UIP holds for short or long term interest rates, we include a short and a long term interest rate. The panel dataset contains observations $i=1, \ldots, N$ countries and at $t=1, \ldots, T_{i}$ time periods. Therefore, the log exchange rate of country $i$ at time $t$ is written as $s_{i t}$. Since we are interested in the change of the exchange rate we calculate first differences as $\Delta s_{i t}=$ $s_{i t}-s_{i, t-1}$. Then $\Delta s_{i t}$ is interpreted as the growth rate of the exchange rate. It is impossible to include the expected exchange rate growth in the empirical analysis because it is unobservable. Instead we use the observable growth rate denoted as $\Delta s_{i t}$ as a proxy for the expectations formed at time $t$. In doing so we assume economic agents have static expectations, i. e. $\mathrm{E}\left(\Delta s_{i t+1}\right)=\Delta s_{i t}$. Although this assumption can be problematic too, we proceed in order to make empirical analysis feasible.

Furthermore, let $\left(r_{l}-r_{l}^{*}\right)_{i t}$ be the long-term interest rate differential, where $r_{l}$ and $r_{l}^{*}$ denote the domestic and foreign long-term rates respectively. Finally, we use $\left(r_{s}-r_{s}^{*}\right)_{i t}$ to denote the short-term interest differential. At best, economic theory predicts that UIP holds in the long-run (see Burda and Wyplosz (1993)). We test this assumption by including short and long-term interest rate differentials in the estimated equation. If the UIP holds in the long-run, we expect $\beta_{1}$ to be positive and close to unity and $\beta_{2}=0$.

Now, we can estimate an equation of the following form:

$$
\Delta s_{i t}=\beta_{0}+\beta_{1}\left(r_{l}-r_{l}^{*}\right)_{i t-1}+\beta_{2}\left(r_{s}-r_{s}^{*}\right)_{i t}
$$

where the interest rate differentials have different time indices to avoid problems with multicollinearity between the two regressors.

\section{The Data}

$z=\operatorname{panstats}(z\{, T\})$

computes summary statistics for the variables 
Before we can estimate equation (3) we have to load the XploRe quantlib metrics for panel data analysis. This is easily be done by typing

library ("metrics")

The complete XploRe code needed for the subsequent analysis is included in

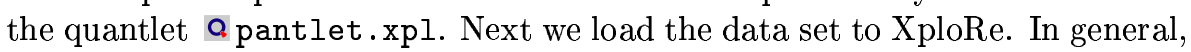
a panel data set is assumed to be ordered by the cross-section units. (If the data are ordered according to the time index, the pansort quantlet can be used to reorganize the data.) That is, the complete data of the first individual is given in the first $T_{1}$ rows, then the data of the second individuals in the rows $T_{1}+1, \ldots, T_{1}+T_{2}$ and so on. If the data set is unbalanced, the first two columns must provide the identification number of the cross-section unit and the time period. If the data are in balanced panel form, it is sufficient to provide the common number of time periods $T$ to assign the data to the cross-section units.

The data matrix must be organized in the following form:

\begin{tabular}{cccccc}
\hline \hline 1 & 2 & 3 & 4 & $\cdots$ & $3+m$ \\
\hline 1 & 1 & $y_{11}$ & $x_{11,1}$ & $\cdots$ & $x_{11, m}$ \\
$\vdots$ & & & & & $\vdots$ \\
1 & $T_{1}$ & $y_{1 T_{1}}$ & $x_{1 T_{1}, 1}$ & $\cdots$ & $x_{1 T_{1}, m}$ \\
2 & 1 & $y_{21}$ & $x_{21,1}$ & $\cdots$ & $x_{21, m}$ \\
$\vdots$ & & & & & $\vdots$ \\
\hline \hline
\end{tabular}

The first and the second column provide the cross-section identification number and the time period respectively. $y_{i t}$ in the third column is the dependent variable, whereas the next $m$ columns contain the explanatory variables. For more details on the panel data set structure needed by XploRe see the XploRe Learning Guide (Section 12.4.1).

Next we load the panel data from the data file uippanel:

z=read ("uippanel.dat")

This example data set has already the appropriate format for the following analysis. To clarify the structure of a XploRe panel data set we have reproduced rows 20 to 25 from the UIP data: 


$\begin{array}{llrrrr}{[20,]} & 1 & 92 & 95.217 & -1.3578 & 2.1699 \\ {[21,]} & 1 & 93 & 97.091 & -1.3401 & 1.3319 \\ {[22,]} & 1 & 94 & 96.962 & -2.7027 & -0.51471 \\ {[23,]} & 1 & 95 & 100 & -1.0097 & -0.20261 \\ {[24,]} & 2 & 73 & 87.671 & 1.2726 & -0.07492 \\ {[25,]} & 2 & 74 & 89.041 & 0.51274 & 0.3582\end{array}$

The first column is the index of a specific country, e.g. 1 corresponds to Austria and 2 to Belgium (see Table 1 for all country codes). The second column contains the year of the observations and hence is the time index. Real effective exchange rate index is given in column three. This index measures the purchasing power relative to the OECD average. The base year of this index is 1995 .

In the fourth column of the data set we find the long-term interest rate differential defined as the difference between the long-term domestic real interest rate and its foreign counterpart. The foreign interest rate corresponds to a weighted average of long-term real interest rates from 17 industrial countries. More precisely, this average is computed from interest rates of the fifteen European Union members, Canada, Japan and the United States left out the country the spread is computed for.

Finally, the fourth column is the short-term real interest spread, i.e. the difference between the domestic short-term interest rate and the foreign short term rate. Again, the foreign interest is constructed by the procedure characterized above.

To detect missing values or potential problems with the data we highly recommend to first compute summary statistics for the panel. In XploRe this is conveniently done by typing

panstats (z)

where $\mathbf{z}$ is the data set. For the UIP data the output turns out to be:

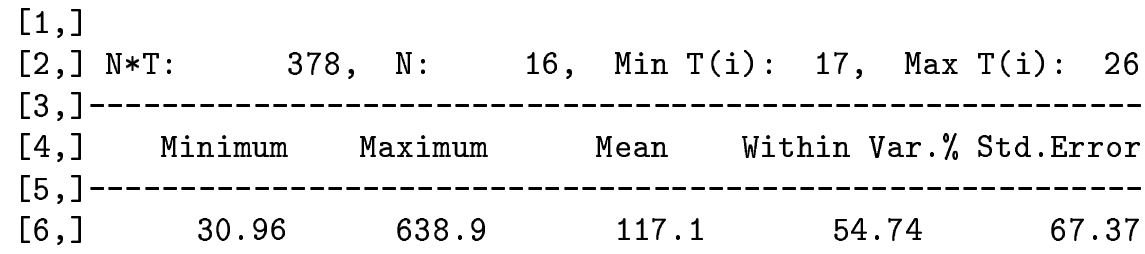




$\begin{array}{llllll}{[7,]} & -13.77 & 9.852 & 0.3543 & 70.82 & 3.082 \\ {[8,]} & -15.13 & 8.324 & 0.2948 & 79.63 & 2.978\end{array}$

$[9$,

The summary table gives an informative overview of the data set properties. First, we can easily see that there are 16 countries included. Next and perhaps more interesting is the fact that the UIP panel is unbalanced. The data set includes at most 26 observations from 1973 to 1998, however, for some countries the interest rate spread is not available for the whole period. These missings have been deleted prior to this analysis such that the resulting data set is unbalanced.

The column "within Var.\%" gives the fraction of variance due to the withingroup deviations. A zero in this column implies that the respective variable is constant over time. This is an important information for the estimation of fixed effects models. Not surprisingly, in our example macroeconomic data set none of the variables is constant over time. Since there are no missings and no other problems with the data, we proceed with a fixed effects model.

\section{A Fixed Effects Model}

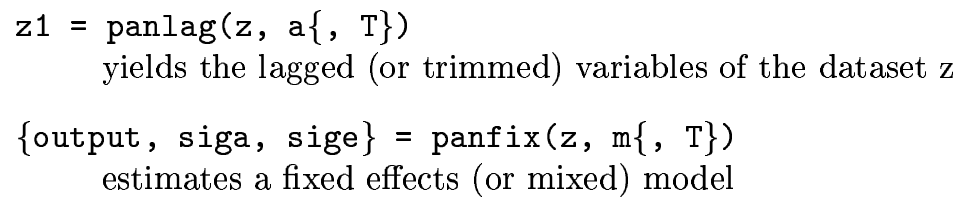

Using notation introduced above we write the modified UIP relation as a fixed effects model of the form:

$$
\Delta s_{i t}=\beta_{0}+\beta_{1}\left(r_{l}-r_{l}^{*}\right)_{i t-1}+\beta_{2}\left(r_{s}-r_{s}^{*}\right)_{i t}+\alpha_{i}+u_{i t}
$$

With the country specific intercept $\alpha_{i}$ it is possible to capture unobservable country specific heterogeneity. In our example we assume that the individual effects $\alpha_{i}$ is deterministic. A comprehensive overview of this model type is given by Hsiao (1986). In a fixed effects model regressors may be correlated with the individual effects, while the error term $u_{i t}$ is uncorrelated across $i$ and $t$ with $E\left(u_{i t}\right)=0, E\left(u_{i t}^{2}\right)=\sigma^{2}$. However, the panfix quantlet does not require such restrictive assumptions. 
In order to estimate equation (4) we need to construct the appropriate variables from variables saved in the original data set. First we use

$$
z[, 3]=\log (z[, 3]) * 100
$$

to compute the logarithm of the exchange rate index and multiply by 100 in order to interpret the result as annual changes in percent. Then we use the quantlet panlag to compute the lagged variables. Specifically the panlag performs the transformation $(1-a) z_{t}-a z_{t-1}$. To obtain the lag of the variable $z_{t}$ we therefore set $a=1$. In general the quantlet is called by

$$
z 1=\operatorname{panlag}(z, a\{, T\})
$$

Using $a=1$ returns a dataset $\mathrm{z} 1$ with the lagged variables of $\mathrm{z}$. As usual, the optional parameter $\mathrm{T}$ is used to indicate the common number of time periods in a balanced dataset.

The command

$$
d z=\operatorname{panlag}(z, 0)-\operatorname{panlag}(z, 1)
$$

stores the first differences of the variables in $z$ in the new dataset $d z$ if $z$ is an unbalanced dataset with the indices for the cross section units and the time periods in the first two columns.

In our case we apply the panlag quantlet as follows:

$$
\begin{aligned}
& \text { y1 }=\operatorname{panlag}(z, 1) \\
& \text { y0=panlag }(z, 0)
\end{aligned}
$$

and compute the relevant data set as:

$$
\mathrm{z} 1=\mathrm{y} 0[, 1: 2] \sim(\mathrm{y} 0[, 3]-\mathrm{y} 1[, 3]) \sim \mathrm{y} 1[, 4] \sim \mathrm{y} 0[, 5]
$$

To estimate the fixed effects model (4) the panfix quantlet is used. Generally, panfix is called as:

$$
\text { \{output, siga, sige }\}=\operatorname{panfix}(z, m\{, T\})
$$


The string output yields the output table of an estimation assuming the first given $\mathrm{m}$ explanatory variables as time varying and correlated with the individual effects. The remaining variables are assumed to be uncorrelated with the individual effect. The common time period $\mathrm{T}$ is included in the list of input parameters if the data is a balanced panel.

For the UIP example we simply type

panfix $(z 1,2)$

Since we have set $m=2$, all regressors are allowed to be correlated with the individual effect $\alpha_{i}$. In this case an within-group estimator is applied. The standard errors of this estimator are estimated in a robust fashion, that is, the standard errors are valid for quite general forms of autocorrelation and heteroskedasticity. The results from panfix are given as:

\begin{tabular}{|c|c|c|c|c|}
\hline 1,$]$ & \multicolumn{4}{|c|}{ 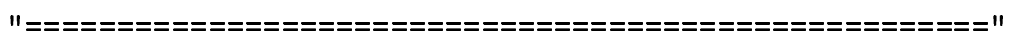 } \\
\hline$[2]$, & \multicolumn{4}{|c|}{ "Fixed-Effect Model: $y(i, t)=x(i, t)$ 'beta+ $a(i)+e(i, t) "$} \\
\hline 3,$]$ & \multicolumn{4}{|c|}{ 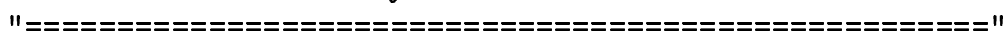 } \\
\hline , 」 & "PARAMETERS & Estimate & robust $\mathrm{SE}$ & \\
\hline , ] & \multicolumn{4}{|c|}{ 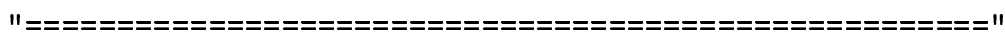 } \\
\hline , 」 & "beta[ 1 ]= & 0.38256 & & $85^{\prime \prime}$ \\
\hline & {$[2]=$} & 0.2 & & $30 "$ \\
\hline , 与 & "CONSTANT = & -1.1781 & 0.6295 & $-1.872^{\prime \prime}$ \\
\hline & \multicolumn{4}{|c|}{ 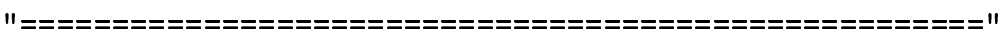 } \\
\hline , ] & "Var. of $a(i)$ : & 6.43 & $e(i, t):$ & $241 "$ \\
\hline & "AR (1) & -val: 0. & & \\
\hline & "F(no eff.) & $\mathrm{p}$-val: 0.00 & e: & $0.2549^{\prime \prime}$ \\
\hline & "LM & p-val: 0.0000 & ke: & -61 \\
\hline
\end{tabular}

The $F$-test clearly rejects the null hypotheses of no effects and hence individual effects $\alpha_{i}$ are significant. However, comparing estimates of $\sigma_{\alpha}^{2}=6.43$ and $\sigma_{u}^{2}=31.24$ it turns out that the remaining error dominates the individual effect.

Nevertheless, the coefficient estimates seem to be reasonable. While $\beta_{2}$ is not significant on conventional significance levels, $\beta_{1}$ is significantly different from zero on the $10 \%$ level. Furthermore the sign of $\beta_{1}$ is in line with expectations. We thus interpret this result as evidence in favor of the UIP. 
A potential problem with the fixed effects estimates is the autocorrelation of the errors. From the panfix output we conclude that there is a significant first order autocorrelation of the residuals. It follows that the standard deviations and $t$-statistics may be biased if they are computed in the usual way. In the panfix quantlet the standard deviations and $t$-statistics are however computed in a robust fashion as suggested by Arellano (1987) so that possible autocorrelation or heteroskedasticity of the errors do not bias the inference. Nevertheless, the estimates may be inefficient and in many applications one is interested to estimate the dynamic relationship explicitely. In the next section we therefore estimate a dynamic panel data model.

\section{A Dynamic Panel Data Model}

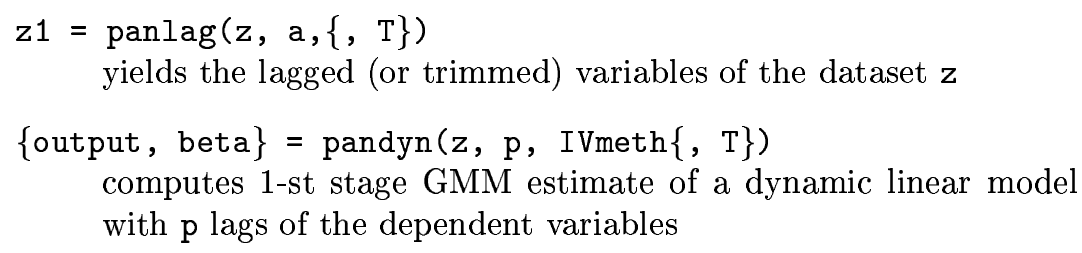

A dynamic panel data model is given by

$$
y_{i t}=\gamma_{1} y_{i, t-1}+\cdots+\gamma_{p} y_{i, t-p}+x_{i t}^{T} \beta+\alpha_{i}+\varepsilon_{i t}
$$

For such a model the within-group estimator is not applicable. Therefore, Arrelano and Bond (1991) suggest to estimate the model using a GMM estimation procedure. The idea is to estimate the differenced model

$$
\Delta y_{i t}=\gamma_{1} \Delta y_{i, t-1}+\cdots+\gamma_{p} \Delta y_{i, t-p}+x_{i t}^{T} \beta+\Delta \varepsilon_{i t}
$$

where $\Delta$ is the difference operator such that $\Delta y_{i t}=y_{i t}-y_{i, t-1}$ by using the instruments:

$$
y_{i, t-2}, y_{i, t-3}, \ldots, y_{i 1}, \Delta x_{i t}
$$

In general, it is possible to construct different GMM estimators using the instruments from (7). Arrelano and Bond (1991) suggest several instrumental variable matrices which are also implemented in XploRe. (For details on the different methods see Xplore Learning Guide, Section 12.5.) In our example 
we have a panel with $T>N$. In this case we only use $y_{i, t-2}, \Delta x_{i, t}$ and $\Delta x_{i, t-1}$ as instruments for lagged differences:

$$
\text { Method 1: }\left(\begin{array}{ccccc}
y_{i p} & y_{i, p+1} & y_{i, p+2} & \cdot & y_{i, T-2} \\
\Delta x_{i, p+1} & \Delta x_{i, p+2} & \Delta x_{i, p+3} & \cdot & \Delta x_{i, T-1} \\
\Delta x_{i, p+2} & \Delta x_{i, p+3} & \Delta x_{i, p+4} & \cdot & \Delta x_{i T}
\end{array}\right) .
$$

To continue our UIP example we rewrite our model in dynamic form as

$$
\Delta s_{i t}=\gamma_{1} \Delta s_{i, t-1}+\beta_{1}\left(r_{l}-r_{l}^{*}\right)_{i, t-1}+\beta_{2}\left(r_{s}-r_{s}^{*}\right)_{i t}+\alpha_{i}+u_{i t} .
$$

We include the one-period lag of the exchange index growth rate as an additional regressor. To estimate equation (8) we use the XploRe quantlet pandyn with the general syntax

$$
\{\text { output, beta }\}=\operatorname{pandyn}(z, p, \operatorname{IVmeth}\{, T\})
$$

where $\mathrm{z}$ is the data set, $\mathrm{p}$ the number of lagged dependent variables, IVmeth the method for constructing the instrument matrix and $\mathrm{T}$ the number of period covered in the data set. Note that the T is only needed if the panel is balanced. For the UIP data we just type

$$
\operatorname{pandyn}(z 1,1,1)
$$

to get the following output table:

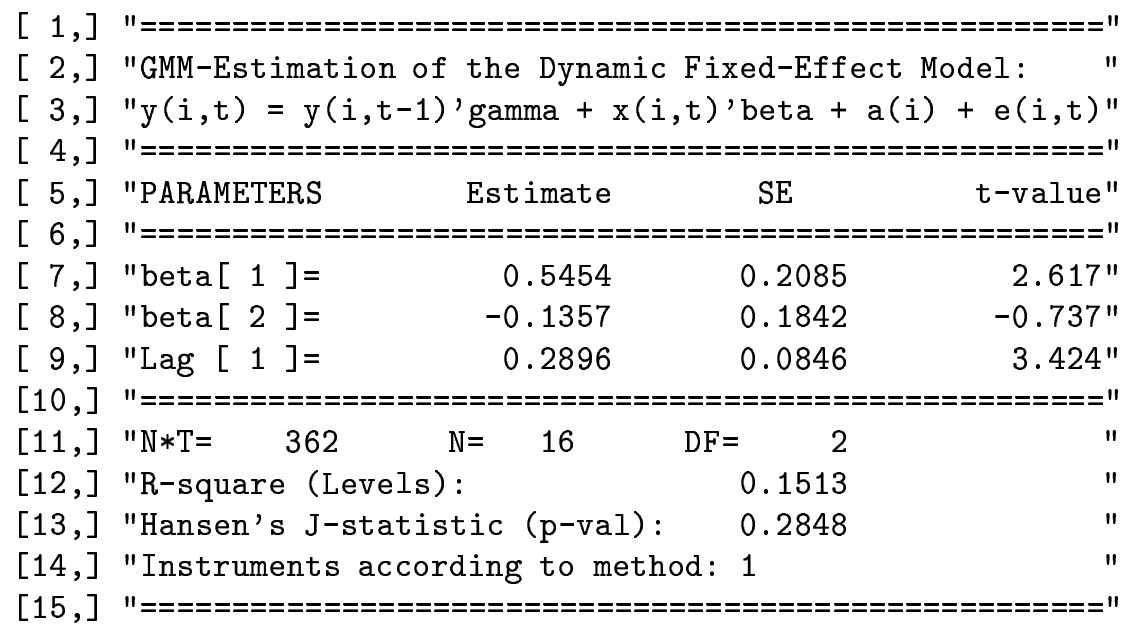


As in the static model, $\beta_{2}$ turns out to be insignificant on conventional levels and in line with results from the static fixed effects model $\beta_{1}$ is now significantly different from zero on the $5 \%$ level. Moreover, the coefficient of the lagged dependent variable $\Delta s_{i, t-1}$ is significant and about the same magnitude as in the test for autocorrelation in the static model. Now that we account for first order autocorrelation the parameters are estimated more precisely. These estimates support the evidence in favor of UIP theory.

\section{Unit Root Tests for Panel Data}

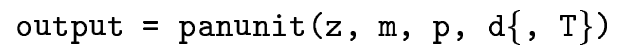
in the data set $\mathrm{z}$ with $\mathrm{p}$ lagged and different deterministic term indicated by $\mathrm{d}$.

In the previous sections we implicitely assumed that real exchange rates $s_{i t}$ are difference stationary variables and the real interest rates are stationary in levels. This assumption is also made by MacDonald and Nagayasu (1999), for example. In the recent literature of dynamic panel data tests have been suggested to test such hypotheses. Following Dickey and Fuller (1979), the unit root hypothesis can be tested by performing the regression:

$$
\Delta y_{i t}=\mu_{i}+\beta_{i} t+\varrho_{i} y_{i, t-1}+\alpha_{i 1} \Delta y_{i, t-1}+\cdots+\alpha_{i p} \Delta y_{i, t-p}+u_{i t}
$$

and testing $\varrho_{i}=0$ for all $i$. The test procedure of Breitung and Meyer (1994) assumes that $\beta_{1}=\cdots=\beta_{N}$ and $\alpha_{1 j}=\cdots=\alpha_{N j}$ for $j=1, \ldots, N$. Thus, as in traditional panel data models, heterogeneity is represented solely by an individual specific intercept. Under this simplifying assumptions a pooled autoregression can be estimated and the lag order can be chosen with respect to the highest significant lag. Therefore, we propose to select the lag order of the model by testing the last lag in the autoregressive specification. This procedure is equivalent to select the lag order by using the highest significant partial autocorrelation. Similarly, the deterministic terms can be selected.

A simple test for the unit root hypothesis is obtained by running the regression

$$
\Delta y_{i t}=\beta t+\varrho\left(y_{i, t-1}-y_{i 1}\right)+\alpha_{1} \Delta y_{i, t-1}+\cdots+\alpha_{p} \Delta y_{i, t-p}+u_{i t}^{*}
$$


The $t$-statistic for $H_{0}: \varrho=0$ is asymptotically standard normal as $N \rightarrow \infty$. Since this procedure is also valid for small $T$ (e.g. $T=10$ ), this test called BM in the panunit output is recommended if only a small number of time periods is available.

Levin and Lin (1993) extend the test procedure to individual specific time trends and short run dynamics. At the first stage the individual specific parameters are "partialled out" by forming the residuals $e_{i t}$ and $v_{i, t-1}$ from a regression of $\Delta y_{i t}$ and $y_{i, t-1}$ on the deterministics and the lagged differences. To account for heteroskedasticity the residuals are adjusted for their standard deviations yielding $\tilde{e}_{i t}$ and $\tilde{v}_{i, t-1}$. The final regression is of the form

$$
\tilde{e}_{i t}=\varrho \tilde{v}_{i, t-1}+\nu_{i t}
$$

If there are no deterministics in the first-stage regressions, the resulting $t$ statistic for the hypothesis $\varrho=0$ is asymptotically standard normally distributed as $T \rightarrow \infty$ and $N \rightarrow \infty$. However, if there is a constant or a time trend in the model, then second-stage $t$-statistic tends to infinity as $T \rightarrow \infty$, even if the null hypothesis is true. Levin and Lin (1993) suggest a correction of the $t$-statistic to remove the bias and to obtain an asymptotic standard normal distribution for the test statistic. Monte Carlo simulations indicate that the test may perform poorly for $p>1$ and, thus, the test should only be used for $p=0$ or $p=1$ (see Im, Pesaran, and Shin (1997) and Breitung (1999)).

Another way to deal with the bias problem of the $t$-statistic is to adopt a different adjustment for the constant and the time trend. The resulting test statistics are called the unbiased Levin-Lin statistic. In the model with a constant term only, the constant can be removed by using $\left(y_{i, t-1}-y_{i 1}\right)$ instead of $y_{i, t-1}$. The first stage regression only uses the lagged differences as regressors. At the second stage, the regression is

$$
\tilde{e}_{i t}=\varrho\left(\tilde{v}_{i, t-1}-\tilde{v}_{i 1}\right)+\nu_{i t}
$$

and the resulting $t$-statistic for $\varrho=0$ is asymptotically standard normal as $T \rightarrow \infty$ and $N \rightarrow \infty$. If there is a linear trend in the model the nuisance parameters are removed by estimating the current trend value by using past values of the process only. Accordingly, the series are adjusted according to

$$
\begin{aligned}
\tilde{e}_{i t}^{*} & =\tilde{e}_{i t}-\tilde{e}_{i 1} \\
\tilde{v}_{i, t-1}^{*} & =\tilde{v}_{i, t-1}-\frac{t-1}{T} \tilde{v}_{i T}
\end{aligned}
$$

Note that $T^{-1} \tilde{v}_{i T}=T^{-1} \sum_{t=1}^{T} \Delta \tilde{v}_{i T}$ is an estimate of the drift parameter. 
Again, the resulting modification yields a $t$-statistic with a standard normal limiting distribution Breitung (1999).

Im, Pesaran, and Shin (1997) further extended the test procedure by allowing for different values of $\varrho_{i}$ under the alternative. Accordingly, all parameters were estimated separately for the cross-section units. Let $\tau_{i}(i=1, \ldots, N)$ denote the individual $t$-statistic for the hypothesis $\varrho_{i}=0$. As $T \rightarrow \infty$ and $N \rightarrow \infty$ we have

$$
N^{-1 / 2} \sum_{i=1}^{N}\left(\tau_{i}-\mu_{\tau}\right) / s_{\tau} \stackrel{d}{\rightarrow} \mathcal{N}(0,1),
$$

where $\mu_{\tau}$ and $s_{\tau}$ is the expectation and standard deviation of the statistic $\tau_{i}$. Im, Pesaran, and Shin (1997) present the mean and variances for a wide range of $T$ and $p$. The quantlet panunit uses estimated values for $\mu_{\tau}$ and $s_{\tau}$ that are obtained from regressions on $\sqrt{T}, T$, and $T^{2}$ and $p$.

Generally, the quantlet computing all these unit root statistics is called as follows:

output $=\operatorname{panunit}(z, m, p, d\{, T\})$

The parameters necessary for computing the statistics are as follows. The parameter $\mathrm{m}$ indicates the column number of the variable to be tested for a unit root. The parameter $\mathrm{p}$ indicates the number of lagged differences in the model. The parameter $\mathrm{d}$ indicates the kind of deterministics used in the regressions. A value of $d=0$ implies that there is no deterministic term in the model. If $d=1$, a constant term is included and for $\mathrm{d}=2$ a linear time trend is included. Finally, if a balanced panel data set is used, the common time period $\mathrm{T}$ is given.

In our application, we test for unit roots in the interest rate differential. The unit root tests for the long-term interest spread (second variable) including a constant and a single lagged difference are obtained using the command

$$
\text { panunit }(z, 2,1,1)
$$

The results can be found in the output table:

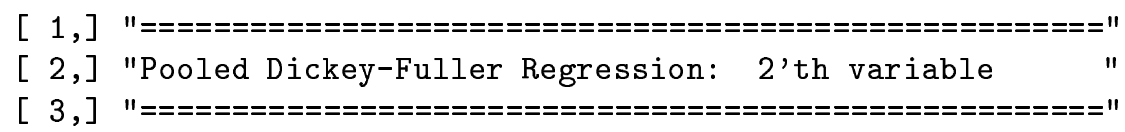




\begin{tabular}{|c|c|c|c|c|c|}
\hline$[4]$, & "PARAMETERS & Esti & imate & robust SE & t-value" \\
\hline$[5]$, & $=========$ & $=====$ & $========$ & $==========$ & $======="$ \\
\hline 6,$]$ & "Lag [1] = & & .2696 & 0.0296 & $-9.117^{\prime \prime}$ \\
\hline 7,$]$ & "Delta[ 1]= & & .0863 & 0.0551 & $-1.566^{\prime \prime}$ \\
\hline 8,$]$ & "const= & & .1109 & 0.1137 & $0.976^{\prime \prime}$ \\
\hline 9,$]$ & "====-====-== & $:======$ & $:=========$ & $=========0$ & $========"$ \\
\hline & "N*T= & $\mathrm{N}=$ & 16 & With cons & ant \\
\hline & "Unit root st & istics: & & & \\
\hline & "STATISTIC & Value & crit. Vall & e $(5 \%)$ & variance" \\
\hline & $"============$ & $=======$ & $:==========$ & $=============$ & $========"$ \\
\hline & "B/M (1994) & -2.453 & -1.65 & 0.000 & $1.000^{\prime \prime}$ \\
\hline & "L/L（1993） & -3.563 & -1.65 & -0.560 & $0.856^{\prime \prime}$ \\
\hline & "mod. L/L & -3.711 & -1.65 & 0.000 & $1.000 "$ \\
\hline & "I/P/S (1997) & -5.313 & -1.65 & -1.493 & $0.756^{\prime \prime}$ \\
\hline
\end{tabular}

All four unit root tests clearly reject the hypotheses of a unit root in the longterm interest spread. Similar result are obtained for the short-term interest differential (not reported). These results are in line with macroeconomic theory on the international term structure.

\section{Conclusions}

We use macroeconomic panel data from 16 countries to estimate a model that enables us to find evidence for the UIP. Interestingly enough, we find strong evidence for UIP when we use long-term interest rate differentials. Moreover, the corresponding short-term interest rate coefficient turns out to be zero. We conclude that UIP does not hold in the short-run. Since the static fixed effects model may be inefficient and biased because of significant first order autocorrelation, we specify a dynamic panel data model, which is in turn estimated by GMM. Results from the dynamic model are in line with what has been found before from the static model.

\section{Macro Data}

The data file uippanel contains an effective exchange rate index, a long term and short term interest rate spread from 16 OECD countries. The data are 


\begin{tabular}{lll}
\hline \hline Column & Type & Description \\
\hline 1 & index & country identification number: \\
& & $1=$ Austria, $2=$ Belgium, $3=$ Canada, $4=$ Germany, \\
& & $5=$ Denmark, $6=$ Spain, $7=$ Finland, \\
& & $8=$ France, $9=$ Great Britain, $11=$ Ireland, \\
& & $12=$ Italy, $13=$ Japan, $15=$ Netherlands, \\
& & $16=$ Portugal, $17=$ Sweden, $18=$ United States \\
2 & metric & time period: years $(73, \ldots, 98)$ \\
3 & metric & real effective exchange rate index, $(1995=100)$ \\
4 & metric & real long-term interest rate differential \\
5 & metric & real short-term interest rate differential \\
\hline \hline
\end{tabular}

Table 1: Description of the data file uippanel.

from the German Bundesbank.

\section{References}

Arellano, M. (1987). Computing Robust Standard Errors for Within-groups Estimators, Oxford Bulletin of Economics and Statistics 49: 431-434.

Arellano, M. and Bond, S. R. (1991). Some Tests of Specification for Panel Data: Monte Carlo Evidence and an Application to Employment Equations, Review of Economic Studies 58: 277-297.

Baltagi, B. H. (1995). Econometric Analysis of Panel Data, Wiley, Chichester.

Breitung, J. (1999). The Local Power of Some Unit Root Tests for Panel Data, SFB 373 Discussion Paper 69/1999.

Breitung, J. and Meyer, W. (1994). Testing for Unit Roots in Panel Data: Are wages on different bargaining levels cointegrated? Applied Economics 26: $353-361$.

Burda, M. C. and Wyplosz, C. (1993). Macroeconomics - A European Text, Oxford University Press, Oxford.

Dickey, D. A. and Fuller, W. A. (1979). Distribution of the Estimates for Autoregressive Time Series With a Unit Root, Journal of the American Statistical Association 74: 427-431. 
Härdle, W., Klinke, S., and Müller, M. (2000). XploRe Learning Guide, Berlin: Springer-Verlag.

Hsiao, C. (1986). Analysis of Panel Data, Cambridge University Press, Cambridge.

Im, K. S., Pesaran, M. H., and Shin, Y. (1997 ). Testing for Unit Roots in Heterogenous Panels, University of Cambridge, revised version of the DAE Working Paper No 9526.

Levin, A. and Lin, C.-F. (1993). Unit Root Tests in Panel Data: Asymptotic and Finite-Sample Properties, University of California San Diego, Unpublished Working Paper (revised).

MacDonald, R. and Nagayasu, J. (1999). The Long-Run Relationship Between Real Exchange Rates and Real Interest Rate Differentials: A Panel Study, WP/99/37, IMF Working Paper. 